\title{
Predictors of aided speech recognition, with and without frequency compression, in older adults.
}

\author{
Rachel J Ellis and Kevin J Munro
}

\section{Linköping University Post Print}

\section{Tweet}

N.B.: When citing this work, cite the original article.

Original Publication:

Rachel J Ellis and Kevin J Munro, Predictors of aided speech recognition, with and without frequency compression, in older adults., 2015, International Journal of Audiology, (54), 7, 46775.

http://dx.doi.org/10.3109/14992027.2014.996825

Copyright: Informa Healthcare

http://informahealthcare.com/

Postprint available at: Linköping University Electronic Press

http://urn.kb.se/resolve?urn=urn:nbn:se:liu:diva-121043 
Ellis \& Munro, Predictors of aided speech recognition

Predictors of aided speech recognition, with and without frequency compression, in older adults

Rachel J. Ellis ${ }^{1,2}$ and Kevin J. Munro ${ }^{1,3}$

${ }^{1}$ School of Psychological Sciences, University of Manchester, UK

${ }^{2}$ Linnaeus Centre HEAD, Swedish Institute for Disability Research, Department of Behavioural Sciences and Learning, Linköping University, Sweden

${ }^{3}$ Central Manchester University Hospitals NHS Foundation Trust, Manchester Academic Health Science Centre, Manchester, U.K.

Key words: Hearing Aids, Frequency Compression, Speech Perception, Cognition, Sensorineural Hearing Loss, Predictors of benefit, older adults

\section{Abbreviations}

TMT-A/B = Trail Making Test A/B

RST $=$ Reading Span Test

$\mathrm{FC}=$ Frequency Compression

Corresponding author: Rachel J. Ellis,

Linnaeus Centre HEAD,

Swedish Institute for Disability Research

Department of Behavioural Sciences and Learning

Linköping University 58183

Sweden

Email: Rachel.Ellis@liu.se 
Ellis \& Munro, Predictors of aided speech recognition

\section{ABSTRACT}

Objective: The aim was to investigate whether cognitive and/or audiological measures predict aided speech recognition, both with and without frequency compression (FC).

Design: Participants wore hearing aids, with and without FCfor a total of 12 weeks (six weeks in each signal processing condition, ABA design). Performance on a sentence-in-noise recognition test was assessed at the end of each six week period. Audiological (severity of high frequency hearing loss, presence of dead regions) and cognitive measures (reading span and trail making test scores) were obtained and assessed as predictors of sentence-in-noise recognition with and without FC enabled.

Study sample: Twelve experienced hearing aid users (aged 65-84 years old) with moderate-to-severe high-frequency hearing loss took part in the study.

Results: The results suggest that both auditory and cognitive factors can be predictive of sentence-innoise recognition with conventional amplification. However, only auditory factors were significantly correlated with the degree of benefit obtained from FC.

Conclusions: The strongest predictor of aided speech recognition, both with and without FC, was high frequency hearing loss. Cognitive performance was also a predictor of benefit from conventional amplification, but not of additional benefit from the use of FC. 
Ellis \& Munro, Predictors of aided speech recognition

Individual differences play a significant role in determining the success of a hearing aid fitting, be that in terms of speech perception scores, hearing aid usage or satisfaction (see for example, Humes, 2007). Determining the specific nature of the individual differences that relate to, and may be predictive of, hearing aid outcome has been the subject of much research. The aim of the present study was to investigate whether cognitive and audiological factors can be used to predict benefit from frequency compression devices in experienced adult hearing aid users.

The contribution of audiological factors as a predictor of speech perception outcomes following amplification is normally limited to a discussion of hearing thresholds. However, a number of other variables may also fall within this category. Specifically, duration of deafness (or auditory deprivation), previous hearing aid use, or the presence of cochlear dead regions.

Unsurprisingly, most studies seeking to explain individual differences in aided speech perception have identified differences in hearing thresholds as the main source of variance (see for example, Humes, 2007; Tun and Wingfield, 1999). However, the influence of hearing thresholds on speech perception outcome seems to be affected by the type of speech test administered. Specifically, audiometric thresholds tend to account for more variance in relatively easy tasks (such as identifying words in a quiet background) than for more complex tests, such as sentence-in-noise recognition, (Humes, 2007). This is supported by the finding that predictions of the speech in noise recognition scores of older adults, based on audiometric factors, normally overestimate performance, whilst speech in quiet predictions are, as a rule, much more accurate (Hargus and Gordon-Salant, 1995).

Thus, once a task becomes more demanding, the influence of audibility on performance is diminished and the influence of cognitive factors become more pronounced. This seems reasonable given that in order to perceive any sound, the signal must first be detected by the ear, transferred successfully to the brain and then interpreted using cognitive skills. If the signal is relatively intact upon reaching the brain (and not degraded to begin with), fewer cognitive resources will be needed to decipher the signal. If, however, the 
Ellis \& Munro, Predictors of aided speech recognition

signal is degraded in some way, be it via a noisy background, an impaired auditory system or a hearing aid processor, more cognitive resources will be required to make sense of the degraded signal.

In order to make use of degraded perceptual cues, one must have the capacity to allocate sufficient cognitive resources to the task at hand. This process is likely to involve a number of specific cognitive abilities, but seems to be most accurately indexed using complex working memory span tests. Working memory (Baddeley and Hitch, 1974) is a limited capacity system used to temporarily store and manipulate incoming information and, to an extent, to allocate attentional resources accordingly. In a review of studies linking cognition and speech in noise perception, measures of working memory span, such as the reading span test (Daneman and Carpenter, 1980; Rönnberg et al, 1989), were found to be the most reliable predictor of test performance once the influence of audibility had been accounted for (Akeroyd, 2008). Lunner (2003), for example, found that, after controlling for the effects of audibility and age, reading span test score was correlated with both aided and unaided recognition of speech in noise.

Whilst it is generally agreed that cognitive factors are more predictive of speech perception performance when the stimulus is degraded in some way, there is some evidence to suggest that the degree to which working memory span predicts speech recognition may be dependent on the degree to which the stimuli differs from phonological representations stored in the long term memory, rather than simply the degradation itself. Rönnberg and colleagues $(2003,2008)$ proposed a working memory model for ease of language understanding. The model suggests that as long as listening conditions are favourable, speech stimuli are implicitly processed and compared to representations stored in the long term memory. If listening conditions are compromised in some way, be it via noise, hearing loss or hearing aid processing, a mismatch may occur between the stimuli being presented and the representation stored in the long term memory. The model suggests that when such a mismatch occurs, explicit storage and processing resources are required and listening becomes more cognitively demanding (Rudner and Rönnberg, 2008). 
Ellis \& Munro, Predictors of aided speech recognition

Rudner et al (2008) found that in a sample of 102 experienced hearing aid users, working memory span scores did indeed show greater correlation with aided speech in noise perception scores when they were tested with novel compression release times (mismatch condition) compared to when they were tested using the compression release time to which they were accustomed (match condition). These results are of particular interest as they demonstrate that the characteristics of the signal processing strategy used in hearing aids can affect the relationship between cognition and speech in noise perception. Lunner (2003) also suggested that it is important to note that the hearing aid processing may in itself be cognitively demanding. This is in agreement with the results reported by Gatehouse et al (2006) and Cox and Xu (2010) who showed that listeners with greater cognitive skills were more likely to benefit from fast acting compression than those with poorer cognitive performance. The implication from the mismatch theory is that the more the hearing aid processing causes the signal to differ from the representation stored in the long term memory, the greater the predictive role of cognition will be.

One method of signal processing that may increase the likelihood of mismatches occurring relative to conventional amplification is non-linear frequency compression (FC). FC is a method of processing an input signal such that information above a specified cut-off point is amplified and compressed in frequency, whilst low and mid frequency information, below the cut-off point, is simply amplified. This method of processing is employed in a range of hearing aids manufactured by Phonak AG (Switzerland).

The results of previous clinical studies of FC hearing aids have shown mixed results with some listeners appearing to receive significant speech perception benefit from FC, whist others obtain no additional benefit above that provided by conventional amplification (see for example: Simpson et al, 2005, 2006; Glista et al, 2009; Wolfe et al, 2010, 2011; Ellis and Munro, under review).

Previous studies investigating FC in listeners with a hearing loss have provided some evidence that age (with children obtaining more benefit than adults) and severity (or configuration) of hearing loss may be predictive of performance with FC (with listeners with poorer thresholds at high frequencies, but not those with sloping severe hearing loss, obtaining the most benefit) on some speech perception measures. 
Ellis \& Munro, Predictors of aided speech recognition

However, cognitive factors have not yet been investigated as predictors of benefit from FC in listeners

with a hearing loss. Arehart et al (2013) report finding a relationship between performance in the reading span test and sentence in noise recognition with different FC settings in listeners with and without HL. It is important to note, however, that in this study FC was used merely as a method to distort the signal and not applied in the same way as in clinical practice. Furthermore, the FC algorithm studied is different to that used in the Phonak range of hearing aids and is not available commercially in any hearing devices. As such, one should be cautious when extrapolating these findings to the speech perception outcomes of listeners with individually determined FC parameters, prescribed in order to reduce the distortion caused by a hearing loss rather than to introduce additional distortion.

The extent to which working memory capacity and executive function predicted sentence in noise performance in listeners with normal hearing, when the stimuli were processed with and without FC, was investigated by Ellis and Munro (2013). The results showed that cognitive scores were predictive of recognition of speech in noise when it had not been processed using FC. The fact that no evidence of a relation between cognition and the perception of frequency compressed speech was observed may have been influenced by the fact that the listeners in this study had no experience of FC. Thus, it is of interest to determine whether, in a sample of experienced hearing aid users, cognitive factors can be used to predict the recognition of frequency compressed sentences in noise after a period of acclimatisation.

Therefore, the aim of the current study was to investigate the extent to which audiometric and cognitive factors predict both speech perception scores with conventional amplification and additional benefit from FC in experienced adult hearing aid users.

\section{METHOD}

\section{Participants}


Ellis \& Munro, Predictors of aided speech recognition

Twelve adults (aged between 65 and 84 years old, median $=75.5$ years old) with symmetrical bilateral moderate-to-severe high-frequency sensorineural hearing loss and $>1$ year hearing aid experience took part in the study. Ethical approval was granted by Greater Manchester West Research Ethics Committee on behalf of the NHS (Approval code: 10/H1014/44).

\section{Table 1 here}

Further details of each participants hearing loss and relevant history are provided in Table 1 and described in greater detail in Ellis and Munro (under review). Hearing thresholds were measured using pure tone audiometry and the presence of dead regions was measured using the TEN test (Moore et al, 2000; 2004). Frequencies between 250 and $8000 \mathrm{~Hz}$ were assessed using a TEN noise level of at least $+10 \mathrm{~dB}$ SL.

\section{Hearing Aid Fitting and Verification}

Participants were provided with Phonak Naida V SP hearing aids for use in the study. The hearings aid were fitted bilaterally and matched to NAL-NL2 prescription targets (Keidser et al, 2011) with frequency compression disabled. The hearing aid fitting and verification procedures used in this study are described in depth in Ellis and Munro (under review).

\section{Study Design}

The results reported in the current paper are a subset of results obtained in a longer trial reported by Ellis and Munro (under review). In summary, the trial took approximately 18 weeks to complete and was split into four parts. A single group design was used. Prior to fitting the frequency compression hearing aids, there were 3 sessions comprising of the initial screening tests and familiarisation with the speech tests. 
Ellis \& Munro, Predictors of aided speech recognition

After fitting the hearing aids, an A-B-A design was used. All participants wore the hearing aids with frequency compression disabled first (for a mean duration of 3.63 weeks, $\mathrm{SD}=0.81$ ), then enabled (for a mean duration of 6.57 weeks, $\mathrm{SD}=0.95$ ) then disabled again (for a mean duration of 3.95 weeks, $\mathrm{SD}=$ 2.75). Thus, the participants wore the devices at home for approximately six to seven weeks with each setting. According to Gatehouse (1992), at least four-six weeks are required to begin to acclimatise to a new hearing aid setting. Participants completed a number of speech tests on six occasions with frequency compression enabled and disabled. All tests were conducted in a quiet room, acoustically representative of a normal living room. Only the results of the speech in noise test obtained in the session following six weeks experience of FC use and the mean of the results obtained after 3 and 6 weeks of experience without FC will be discussed in this article. The remaining results are discussed in a paper on benefit from FC and acclimatisation to FC respectively (Ellis and Munro, in press). Participants also completed the Reading Span Test and the Trail Making Tests A and B on two occasions, once at the first testing session and once at the penultimate testing session. The mean of the scores obtained on these two occasions were calculated.

\section{Outcome measures}

\section{Sentence-in-Noise recognition}

Participants were presented with lists of 10 Institute of Electrical and Electronics Engineers (IEEE) sentences (Rothauser et al, 1969; for example, 'the last switch cannot be turned off') spoken by a female talker and presented in a background of 20 talker babble noise (Auditec, St Louis, USA). The sentences were presented via a loudspeaker situated at head height one meter from the listener at zero degrees azimuth. The level at which the sentences were presented was kept constant throughout each list, however, the level of the noise varied from +15 to $-12 \mathrm{~dB}$ in a fixed order, one sentence at each SNR, similar to the method recommended by Wilson et al (2007). Sentences were presented at 55 dB SPL for 
Ellis \& Munro, Predictors of aided speech recognition

all participants with the exception of one (participant 008) who was unable to complete the task at this

level. For this listener, sentences were presented at $65 \mathrm{~dB}$ SPL. Participants were asked to repeat each sentence back to the experimenter and the percentage of keywords ( 5 per sentence) correctly recognised was calculated. Three sentence lists were administered on each occasion of testing. The same sentences lists were used for all participants but were randomly allocated to different test conditions.

\section{Cognitive measures}

Participants completed the Reading Span Test (RST) and the Trail Making Tests (TMT) A and B on two occasions, once at the first testing session and once at the penultimate testing session.

The Reading Span Test (Daneman and Carpenter, 1980; Rönnberg et al, 1989) consists of a sequence of sentences in blocks of 3-6. Sentences were presented to the participant, word by word, on a computer screen at the rate of one word every $800 \mathrm{~ms}$. Immediately after reading each sentence, the participant must state whether the sentence made sense giving a yes-no response (processing component). At the end of each block, participants were asked to recall either the first or last word of every sentence in that group (storage component). The order in which participants were asked to recall either first or last words of each sentence was randomized. In the first trial, three sentences were presented. In each subsequent trial the number of sentences presented was increased by one, up to six sentences in the final trial. Upon completion of the test, the percentage of words correctly recalled was then calculated. The percentage of errors made in the processing component of the task was also calculated.

The Trail Making Tests A and B (TMT-A/B, Reitan, 1958, 1992) are paper and pencil based tasks thought to index processing speed and executive function. The TMT was included in the study as it has been shown that performance on the TMT may predict the ability of listeners with a hearing loss to perceive speech in a novel accent (Adank and Janse, 2010). As frequency compression alters the spectral 
Ellis \& Munro, Predictors of aided speech recognition

relationship between speech components, perception of speech processed in this way may require similar skills to those needed to perceive speech in a novel accent. The participants' task was to join together a series of points on a sheet of paper in the correct order without allowing their pencil to leave the page. In the TMT-A, the points are labelled from 1-25, the task being to join the points in the correct numerical order. In the TMT-B, the points are labelled 1-12 and A-L and the participants' task was to join the points in the correct alphabetical and numerical order, alternating between the two (for example, 1-A-2-B-3-C and so on). Prior to completing the TMT tests, the participants were given a sample task on which to practice. Performance on the task was assessed by measuring the total time taken to complete each task. If the participant made a mistake, the experimenter pointed out their error and asked the participant to correct it. Errors were penalized only in that they increased the time taken to complete the task.

Participants were aware that the time taken to complete the task was the outcome measure of interest.

\section{Analysis}

Pearson's correlation coefficient was calculated to investigate the relationship between possible predictors of benefit from frequency compression and scores on the sentence recognition in noise (SIN) test. Predictors were correlated with both the mean percentage correct SIN score without FC and with the additional benefit obtained from frequency compression after 6 weeks use (mean SIN \% correct at FC6 mean SIN \% correct without FC). Potential audiological predictors included mean high frequency pure tone threshold (mean of thresholds at 2, 4, 6 and $8 \mathrm{kHz}$ ) in the better ear (HFPTA) and the presence of dead regions (DRs). The influence of HFPTA was partialled out for all subsequent correlations. Partial correlation was achieved using a general linear model in which the variable of interest (in this case, HFPTA) is correlated with variable A, which is then recalculated so as to remove the effects of HFPTA. The recalculated variable A A is then correlated with variable B to give the correlation between variables A and B with the effects og HFPTA controlled for. As a result of these analyses, some of the axes in the included figures contain negative numbers, when this would not intuitively seem to make sense. 
Ellis \& Munro, Predictors of aided speech recognition

The presence of DRs was coded as a dichotomous variable (where $0=$ no DRs and $1=$ one or more DRs in at least one ear), therefore a point biserial correlation analysis was conducted. Possible cognitive predictors included mean percentage correct on the RST, mean percentage error rate on the RST, mean TMT-A score and mean TMT-B score. Despite the multiple correlations, a Bonferroni correction was not applied as it was felt that the increase in the risk of a Type 2 error occurring outweighed the potential benefits of reducing the risk of a Type 1 error (see Cabin and Mitchell, 2000 for a discussion of when to use the Bonferroni correction).

\section{RESULTS}

\section{Audiological Predictors}

HFPTA

Inspection of the scatterplots shown in the left hand column of Figure 1 indicate a negative correlation between HFPTA and mean SIN scores without FC (upper panel) and a positive correlation with benefit obtained from FC (lower panel). These correlations are both statistically significant, albeit borderline in relation to benefit from FC (No FC: $r=-0.92, p<0.001$; benefit from FC: $r=0.59, p=0.045$ ).

\section{Figure 1 here}

Presence of DRs

The scatterplots shown in the right hand column of Figure 1 suggest that there is no relationship between the presence of DRs and performance in the SIN test either with (lower panel) or without (upper panel) FC. The analysis confirmed that these correlations are not statistically significant (No FC: $r=-0.03, p=$ 0.925; benefit from FC: $\mathrm{r}=0.13, \mathrm{p}=0.705)$. 
Ellis \& Munro, Predictors of aided speech recognition

\section{Cognitive Predictors}

RST: \% Correct Recall

The mean RST \% correct was 29 (SD = 11.09). An inspection of the scatterplots presented in the left hand column of Figure 2 suggest a trend for a positive relationship between RST \% correct scores and performance on the SIN test without FC (upper panel). However, there is no evidence of a relationship between RST \% correct recall and benefit obtained from FC (lower panel). The results of the statistical analyses show that neither of these correlations reaches statistical significance $($ No FC: $\mathrm{r}=0.53, \mathrm{p}=$ 0.094; benefit from FC: $\mathrm{r}=-0.17, \mathrm{p}=0.625)$.

Figure 2 here

RST: \% Processing Errors

The mean percentage of processing errors in the RST was $6.56(\mathrm{SD}=6.53)$. The scatterplots displayed in the right hand column of Figure 2 indicate a negative correlation between RST processing error rate and SIN scores without FC (upper panel) and a positive correlation with SIN scores with FC (lower panel). The results of the statistical analyses indicate that only the correlation between RST error rate and SIN without FC is borderline statistically significant (No FC: $r=-0.60, p=0.050$; benefit from FC: $r=0.34, p$ $=0.306)$.

TMT-A 
Ellis \& Munro, Predictors of aided speech recognition

The mean time taken to complete the TMT-A was 42.92 seconds ( $\mathrm{SD}=30.86$ ). The scatterplots shown in the left hand column of Figure 3 indicate a negative relationship between TMT-A scores and performance in the SIN test without FC. No clear evidence of a relationship between TMT-A score and benefit obtained from FC is apparent in the scatterplot presented in the lower panel of the left hand column of Figure 3. The results of the statistical analyses confirm that only the correlation between TMT-A scores and performance on the SIN test without FC is statistically significant (No FC: $r=-0.69, p=0.018$; benefit from FC: $r=0.28, p=0.413$.

Figure 3 here

TMT-B

The mean time taken to complete the TMT-B was 95.04 seconds (SD = 42.56). The scatterplots displayed in the right hand column of Figure 3 indicate a negative relationship between TMT-B scores and performance in the SIN test without FC (upper panel) with no clear evidence of a relationship between TMT-B scores and benefit obtained from FC (lower panel). The results of the statistical analysis indicate that only the correlation between TMT-B scores and performance on the SIN test without FC is statistically significant (No FC: $r=-0.67, p=0.026$; benefit from FC: $\mathrm{R}=0.06, \mathrm{P}=0.851$ ).

\section{DISCUSSION}

The results of the study indicate that, based on the factors investigated, both HFPTA and cognitive function can be used to predict speech recognition outcomes from conventionally amplified speech. However, cognitive performance did not significantly predict additional benefit from FC. This pattern of results is similar to those observed in a study investigating the role of cognition as a predictor of frequency compressed speech recognition in listeners with normal hearing (Ellis and Munro, 2013). 
Ellis \& Munro, Predictors of aided speech recognition

Whilst HFPTA did predict additional benefit from FC, this finding should be considered cautiously given the heterogeneous nature of the sample group.

The observation that high frequency pure tone average loss is predictive of benefit from frequency compression is consistent with results reported by Glista et al (2009). However, it should be noted that in the present study, this result is likely to have been disproportionately influenced by one particular case (evidenced by a Cook's distance value of 1.49, above the cutoff of 1 recommended by Cook and Weisberg, 1982), the participant with the poorest hearing thresholds. This is not to suggest that the results are invalid, only that they should be interpreted cautiously. The findings of both the present study and that conducted by Glista et al (2009) indicate that listeners with greater high frequency losses obtain more benefit from frequency compression than those with less severe high frequency losses. This suggests that benefit from frequency compression is related to the increased audibility of high frequency sounds resulting from this signal processing method.

Once the effect of high frequency hearing loss was partialled out, the presence of dead regions did not predict sentence-in-noise recognition with or without FC enabled. This is in agreement with results reported by Dillon et al (2011), who found that once the effect of hearing loss had been accounted for, dead regions had little effect on the intelligibility of conventionally amplified speech. However, the analysis in the present study was based simply on whether there was evidence of at least one dead region or not. The edge frequency and width of the dead regions was not taken into account. It is perfectly plausible that these factors may need to be considered when selecting the frequency compression cut-off point. Research has suggested that providing amplification at frequencies over $70 \%$ above the edge frequency of a dead region may be of limited benefit to listeners (Vickers et al, 2001). It may therefore be the case that compressing high frequency information into this region is also of limited benefit to the listener. 
Ellis \& Munro, Predictors of aided speech recognition

The results indicate that, once the effect of high frequency hearing loss has been partialled out, cognitive performance can be used to predict sentence-in-noise recognition without frequency compression. Specifically, results in the TMT-A and B tests, along with the percentage of processing errors made in the RST were related to performance in the speech in noise test. Listeners who obtained better scores in the cognitive tests also tended to achieve higher scores in the sentence recognition task. This finding is consistent with the results of previous studies of sentence recognition scores (see Akeroyd, 2008 for a review). However, the percentage recalled correct obtained in the RST, a measure that has often been shown to correlate with aided speech perception scores (see for example, Lunner, 2003), was not predictive of either performance without frequency compression or additional benefit obtained from it. It should be noted that a positive trend between percentage correct on the RST and sentence recognition without frequency compression is evident, however, this relationship did not reach statistical significance.

The percentage correct score on the reading span test is normally seen as a reflection of working memory span whilst the TMT-A and B are generally seen as indices of executive function and task switching abilities. One may also consider performance in the RST, particularly in respect to error rate, to be an index of executive function (see for example, Engle, 2002). Therefore the results of the current study provide some evidence that it may not be working memory span, per se, but rather executive functioning, that is predictive of speech in noise recognition. This seems logical, given that the ability to efficiently perceive speech in noise depends on the capacity to focus attention on the useful parts of the signal, and away from the competing noise, an ability governed by executive function. That the TMT-A and B were shown here to be predictive of aided speech in noise recognition (albeit without frequency compression) may be of clinical interest. These tests require no special equipment and take only minutes to administer thus making them a practical option to administer in a clinical, as well as a research, setting. However, additional research is needed to further investigate the relationship between executive function and speech perception. 
Ellis \& Munro, Predictors of aided speech recognition

The fact that none of the cognitive measures correlated with additional benefit derived from frequency compression on speech recognition scores may be considered in light of the working memory model of ease of language understanding proposed by Rönnberg and colleagues (2003, 2008, 2013; Rudner and Rönnberg, 2008). According to the model, cognitive resources are taxed when speech stimuli differ from representations stored in the long term memory. It may be that as changes introduced by frequency compression are concentrated only at the high frequencies, the differences between sentences that have been compressed in frequency and those that have not are not large enough to require additional cognitive resources. Furthermore, it is also possible that frequency compression actually makes speech more similar to the representations stored in the long term memory than conventional amplification, depending on the duration and severity of hearing loss (for example, in cases where frequency compression makes the otherwise inaudible high frequency parts of a phoneme audible with a minimal amount of spectral distortion).

Whilst the sample used in this study was small, there was sufficient power to detect that high frequency PTA, TMT-A score, TMT-B score and RST \% errors significantly predicted speech recognition without FC (all except the RST \% error retaining their significance even after Bonferroni correction). We suggest that this shows the study has sufficient power to detect these effects, and, that the fact that the cognitive predictors were not associated with benefit from FC is a genuine result, not one arising from a lack of power. In order to investigate whether correlations between each of the cognitive tests and performance without FC were significantly different to the correlations with the degree of additional benefit obtained from the use of FC, we conducted a series of Steiger's Z tests. The results indicate that the TMT-B test may be particularly sensitive to predicting outcomes without FC yet not the degree of additional benefit obtained from the use of FC (Steiger's $Z=-2.27, p<.05$ ).

\section{Conclusions}


Ellis \& Munro, Predictors of aided speech recognition

The results of the study indicate that severity of high frequency hearing loss can be used to predict sentence in noise recognition with conventional amplification and, to some extent, the degree of additional benefit conferred by the use of frequency compression. However, only benefit from conventional amplification could be predicted from cognitive performance. The finding that benefit from FC is not predicted by performance on cognitive tests used in this study suggests that cognitive ability, at least in terms of the skills indexed by the tests administered in this study, does not need to be considered when assessing the potential candidacy for FC. The clinical implication of this finding is that FC may provide additional benefit to that conferred by conventional amplification, even to less cognitively able patients. The findings also suggest that tests of executive function may be useful in a clinical setting with regard to their value in predicting speech perception outcomes without frequency compression.

\section{ACKNOWLEDGEMENTS}

This study was funded by an ESRC CASE PhD studentship in conjunction with Phonak AG. The authors are grateful to the participants for donating their time and to Catherine Siciliano, Kathryn Hopkins, Richard Baker and Michael Akeroyd for helpful comments on an earlier draft of this paper. The authors would also like to thank Kathryn Lewis and staff at the Audiology department at Withington Community Hospital, Manchester for help recruiting participants, Keith Wilbraham for technical assistance, Anna Pepler for clinical assistance and Andrea Simpson for her help in getting the project started.

Declaration of interest: The authors report no conflict of interest. The authors alone are responsible for the content and writing of the paper. 
Ellis \& Munro, Predictors of aided speech recognition

\section{REFERENCES}

Adank, P. \& Janse, E. (2010) Comprehension of a novel accent by young and elderly listeners. Psychol Aging, 25, 736-740.

Akeroyd, M.A. (2008) Are individual differences in speech reception related to individual differences in cognitive ability? A survey of twenty experimental studies with normal and hearingimpaired adults. Int J Audiol, 47, S53-S71.

Arehart, K. H., Souza, P., Baca, R. \& Kates, J. M. (2013) Working memory, age, and hearing loss: Susceptibility to hearing aid distortion. Ear Hear, 34, 251-260.

Baddeley, A.D. \& Hitch, G. J. (1974) Working memory, in G.H. Bower (Ed.), The Psychology of Learning and Motivation: Advances in Research and Theory (volume 8), pp. 47-90, New York: Academic Press.

Cabin, R. J. \& Mitchell, R. J. (2000) To Bonferroni or not to Bonferroni : When and how are the questions. Bull Ecol Soc Am, 81, 246-248.

Cook, D. R. \& Weisberg, S. (1982) Residuals and influence in regression. New York : Chapman and Hall.

Cox, R. M. \& Xu, J. (2010) Short and long compression release times: Speech understanding, real world preferences, and association with cognitive ability. J Am Acad Audiol, 21, 121-138.

Daneman, M \& Carpenter, P.A. (1980) Individual differences in working memory and reading, $J$ Verbal Learning Verbal Behav, 19, 450 - 466.

Dillon, H., Keidser, G., Ching, T. Y. C. et al (2011) The NAL-NL2 prescription procedure. Phonak Focus 40, Switzerland: Phonak AG.

Ellis, R. J. \& Munro, K. J. (in press) Benefit from, and acclimatisation to, frequency compression hearing aids in experienced adult hearing aid users. Int J Audiol

Ellis, R. J. \& Munro, K. J. (2013) Does cognitive function predict frequency compressed speech 
Ellis \& Munro, Predictors of aided speech recognition

recognition in listeners with normal hearing and normal cognition? Int J Audiol, 52, 14-22.

Engle, R. W. (2002) Working memory capacity as executive attention, Curr Dir Psychol Sci, 11, 19 - 23.

Gatehouse, S. (1992) The time course and magnitude of perceptual acclimatization to frequency responses: Evidence from monaural fitting of hearing aids. J Acoust Soc Am, 92, 12581268.

Gatehouse, S., Naylor, G. \& Elberling, C. (2006b) Linear and nonlinear hearing aid fittings - 2. Patterns of candidature. Int J Audiol, 45, 153-171.

Glista, D., Scollie, S., Bagatto, M., Seewald, R., Parsa, V. et al (2009) Evaluation of nonlinear frequency compression: Clinical outcomes. Int J Audiol, 48, 632-644.

Hargus, S. E. and Gordon-Salant, S. (1995) Accuracy of speech intelligibility index predictions for noisemasked young listeners with normal hearing and for elderly listeners with hearing impairment. J Speech Lang Hear Res, 38, 234-243.

Humes, L. E. (2007) The contributions of audibility and cognitive factors to the benefit provided by amplified speech to older adults. J Am Acad Audiol, 18, 590-603

Keidser, G., Dillon, H. R., Flax. M., Ching, T. \& Brewer, S. (2011) The NAL-NL2 prescription procedure. Audiol Res doi:10.4081 /audiores.2011.e24

Lunner, T. (2003) Cognitive function in relation to hearing aid use. Int J Audiol, 42, S49-58.

Moore, B. C. J., Huss, M., Vickers, D. A., Glasberg, B. R. \& Alcántara, J. I. (2000) A test for the diagnosis of dead regions in the cochlea. Br J Audiol, 34, 205-224.

Moore, B. C. J., Glasberg, B. R. \& Stone, M. A. (2004) New version of the TEN test with calibrations in dB HL. Ear Hear, 25, 478-487.

Reitan, R. M. (1958) Validity of the Trail Making test as an indicator of organic brain damage. Percept Mot Skills, 8, 271-276. 
Ellis \& Munro, Predictors of aided speech recognition

Reitan, R. M. (1992) Trail Making test: Manual for administration and scoring. Arizona: Reitan

Neuropsychology Laboratory.

Rönnberg J. (2003) Cognition in the hearing impaired and deaf as a bridge between signal and dialogue: A framework and a model. Int J Audiol, 42, s68-s76.

Rönnberg J., Arlinger S., Lyxell B. \& Kinnefors, C. (1989) Visual evoked potentials: Relations to adult speechreading and cognitive function. J Speech Lang Hear Res, 32, 725-735.

Rönnberg, J., Lunner, L., Zekveld, A., Sörqvist, P., Danielsson, H. et al (2013) The Ease of Language Understanding (ELU) model: theoretical, empirical and clinical advances. Front Syst Neurosci, 7:31. doi: 10.3389/fnsys.2013.0003

Rönnberg J., Rudner, M., Foo, C. \& Lunner, T. (2008) Cognition counts: A working memory system of ease of language understanding (ELU). Int J Audiol, 47, s99-s105.

Rothauser, E. H., Chapman, W. D., Guttman, N., Nordby, K. S., Silbiger, H. et al (1969) IEEE recommended practice for speech quality measurements. IEEE Trans Acoust,17, 227-246.

Rudner, M. \& Rönnberg J. (2008) The role of the episodic buffer in working memory for language processing. Cogn Pro, 9, 19-28.

Rudner, M., Foo, C., Sundewall-Thorén, E., Lunner, T. \& Rönnberg, J.(2008) Phonological mismatch and explicit cognitive processing in a sample of 102 hearing aid users. Int J Audiol, 47, S91-S98.

Saunders, G. H. \& Cienkowski, K. M. (1997) Acclimatization to hearing aids. Ear Hear, 18, 129-139.

Simpson, A., Hersbach, A. A. \& McDermott, H. J. (2005) Improvements in speech perception with an experimental nonlinear frequency compression hearing device. Int J Audiol, 44, 281-292.

Simpson, A., Hersbach, A. A. \& McDermott, H. J. (2006) Frequency-compression outcomes in listeners with steeply sloping audiograms. Int J Audiol, 45, 619-629.

Tun, P. A. \& Wingfield, A. (1999) One voice too many: Adult age differences in language processing 
Ellis \& Munro, Predictors of aided speech recognition

with different types of distracting sounds. J Gerontol Psychol Sci, 54, 317-327.

Vickers, D. A., Moore, B. C. J. \& Baer, T. (2001) Effects of low-pass filtering on the intelligibility of speech in quiet for people with and without dead regions at high frequencies. J Acoust Soc Am, 110, 1164-1175.

Wilson, R. H., McArdle, R. A. \& Smith, S. L. (2007) An evaluation of the BKB-SIN, HINT, QuickSIN and WIN materials on listeners with normal hearing and listeners with hearing loss. $J$ Speech Lang Hear Res, 50, 844-856.

Wolfe, J., John, A., Schafer, E., Nyffeler, M., Boretzki, M. et al (2010) Evaluation of nonlinear frequency compression for school-age children with moderate to moderately severe hearing loss. J Am Acad Audiol, 21, 618-628.

Wolfe, J., John, A., Schafer, E., Nyffeler, M., Boretzki, M. et al (2011) Long-term effects of non-linear frequency compression for children with moderate hearing loss. Int J Audiol, 50, 396-404. 
Ellis \& Munro, Predictors of aided speech recognition

\section{LIST OF TABLES}

Table 1. Characteristics of the participants' hearing loss, previous hearing aid use and the non-linear frequency compression settings used in the trial. 
Ellis \& Munro, Predictors of aided speech recognition

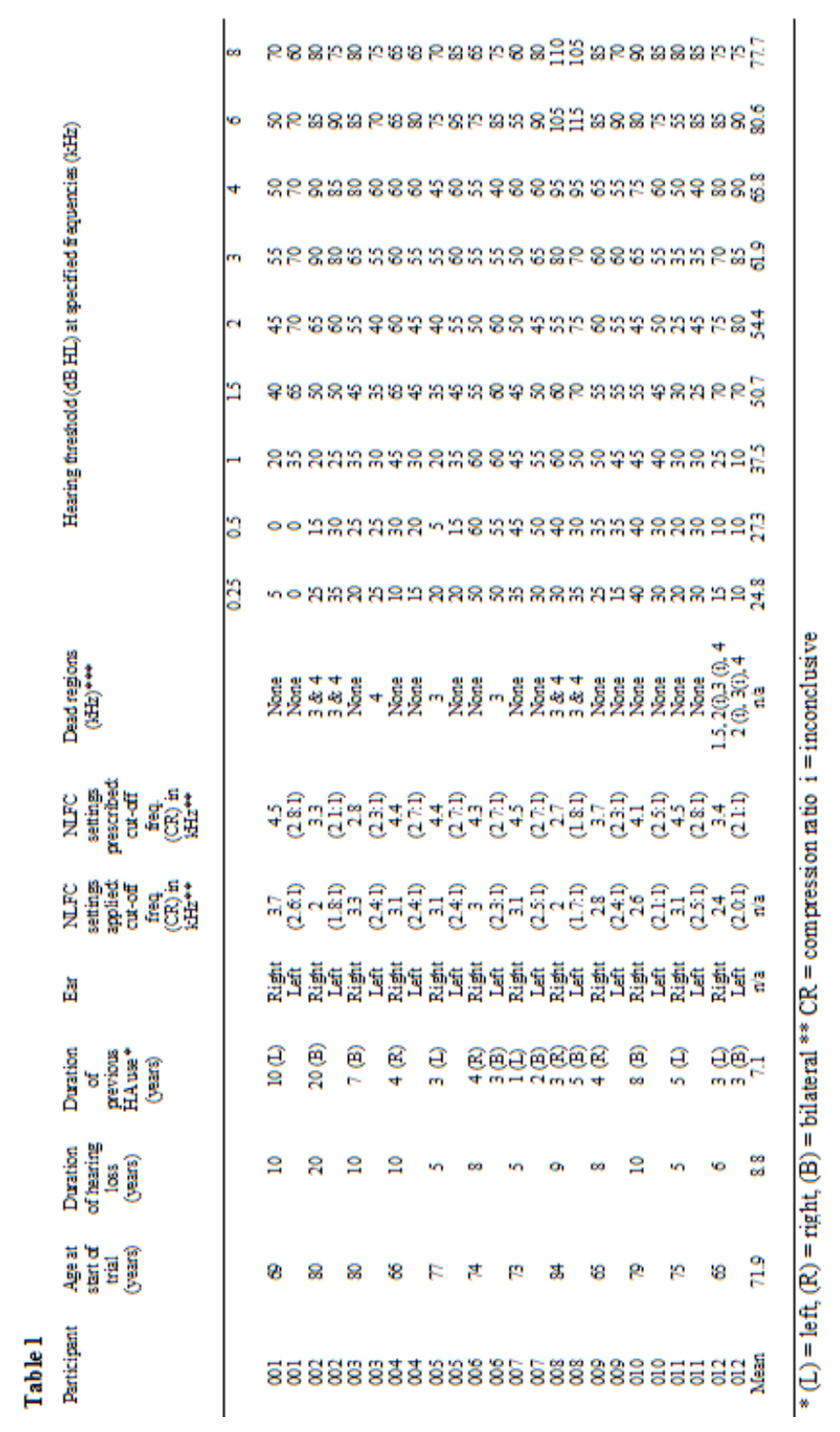


Ellis \& Munro, Predictors of aided speech recognition

\section{LIST OF FIGURES}

Figure 1. Correlations between High Frequency Pure Tone Average (HFPTA, 2,4,6 and 8 kHz) in the better ear and mean percentage correct in the sentence-in-noise test without frequency compression (top left panel) and with benefit obtained from frequency compression (bottom left panel). Partial correlations (with HFPTA controlled for) between the presence of dead regions and the mean percentage correct in the sentence-in-noise test without frequency compression (top right panel) and with benefit obtained from frequency compression (bottom right panel).

Figure 2. Partial correlations (with HFPTA controlled for) between the mean percentage correct obtained on the RST and the mean percentage correct in the sentence-in-noise test without frequency compression (top left panel) and with benefit obtained from frequency compression (bottom left panel). Partial correlations (with HFPTA controlled for) between the mean percentage of errors obtained on the RST with the mean percentage correct recall in the sentence-in-noise test without frequency compression (top right panel) and with benefit obtained from frequency compression (bottom right panel).

Figure 3. Partial correlations (with HFPTA controlled for) between the mean TMT-A scores and the mean percentage correct in the sentence-in-noise test without frequency compression (top left panel) and with benefit obtained from frequency compression (bottom left panel). Partial correlations (with HFPTA controlled for) between the mean TMT-B scores with the mean percentage correct in the sentence-innoise test without frequency compression (top right panel) and with benefit obtained from frequency compression (bottom right panel). 
Ellis \& Munro, Predictors of aided speech recognition Figure 1
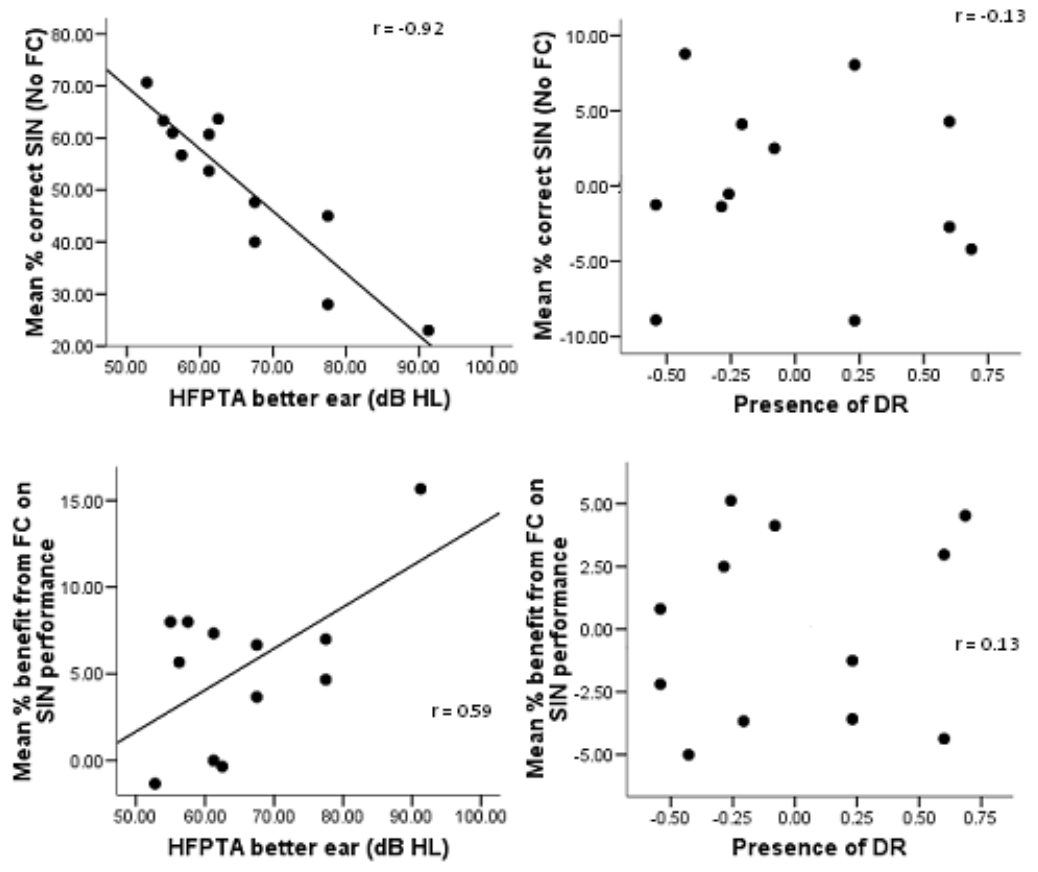
Ellis \& Munro, Predictors of aided speech recognition Figure 2
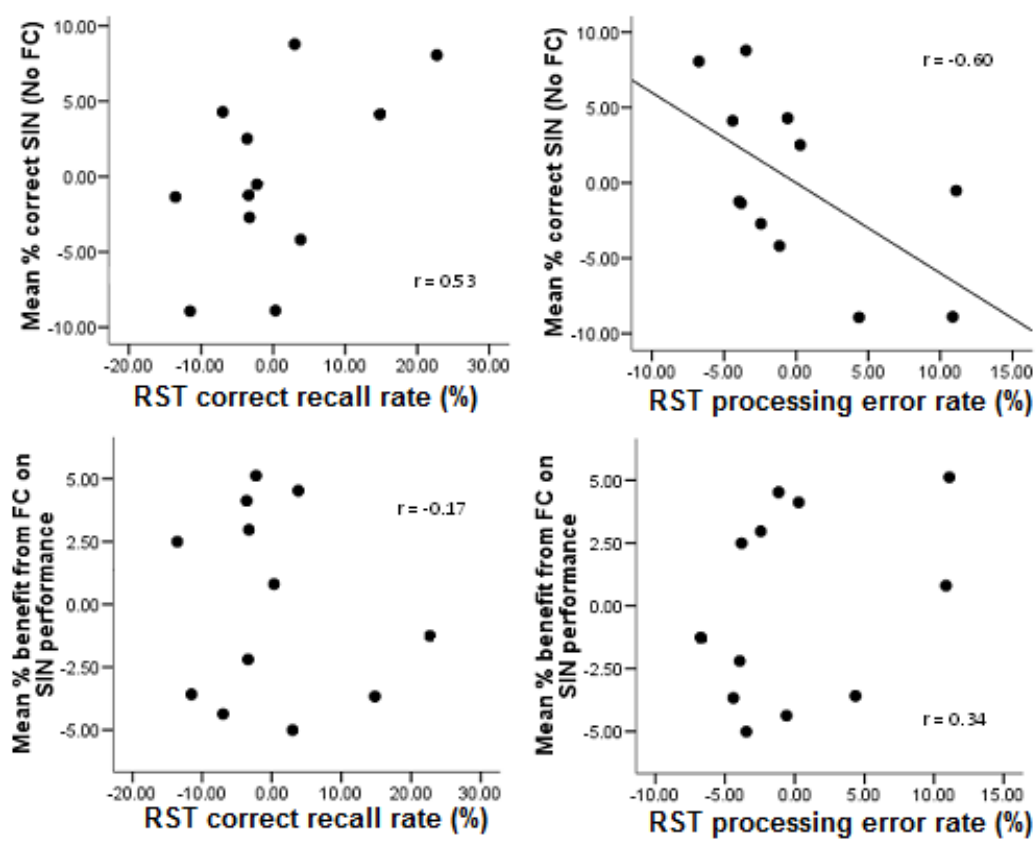
Ellis \& Munro, Predictors of aided speech recognition Figure 3
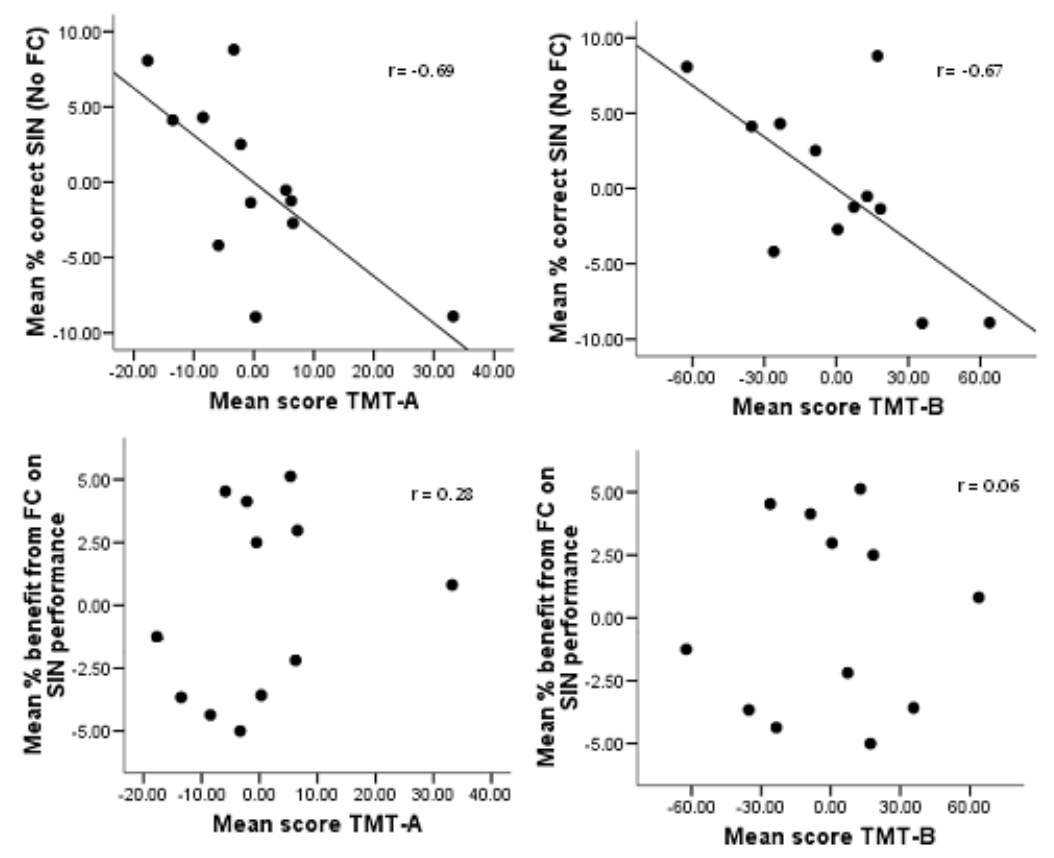
Ellis \& Munro, Predictors of aided speech recognition 